\title{
Preservation of Small Extracellular Vesicle in Gelatin Methacryloyl Hydrogel Through Reduced Particles Aggregation for Therapeutic Applications
}

\author{
Kelun $\mathrm{Wu}^{1-3}$ \\ Chuan $\mathrm{He}^{\mathrm{I}-3}$ \\ Yue $\mathrm{Wu}^{1-3}$ \\ Xiaojie Zhou ${ }^{1-3}$ \\ Pan Liu ${ }^{1-3}$ \\ Wei Tang ${ }^{1,3}$ \\ Mei Yu (D) ${ }^{1,2}$ \\ Weidong Tian ${ }^{1-3}$
}

'State Key Laboratory of Oral Disease \& National Clinical Research Center for Oral Diseases \& National Engineering Laboratory for Oral Regenerative Medicine, West China School of Stomatology, Sichuan University, Chengdu, People's Republic of China;

${ }^{2}$ Engineering Research Center of Oral Translational Medicine, Ministry of Education, West China School of Stomatology, Sichuan University, Chengdu, People's Republic of China; ${ }^{3}$ Department of Oral and Maxillofacial Surgery, West China Hospital of Stomatology, Sichuan University, Chengdu, 610065, Sichuan, People's Republic of China

Correspondence: Mei Yu State Key Laboratory of Oral Disease \& National Clinical Research Center for Oral Diseases \& National Engineering Laboratory for Oral Regenerative Medicine, West China School of Stomatology, Sichuan University, Chengdu, People's Republic of China Email yumei925@hotmail.com

Wei Tang

Department of Oral and Maxillofacial Surgery, West China Hospital of Stomatology, Sichuan University, Chengdu, People's Republic of China Tel/Fax +86-28-85503499

Email mydrtw@vip.sina.com
Purpose: Small extracellular vesicles (sEV) play an irreplaceable role in cell-cell communication. However, sEV in solution aggregate with each other during preservation, leading to impairment of the structures, contents, and functions of sEV. Therefore, there is a need to develop an optimal preservation method that combines high recovery rate, low cost, convenience, and easy-transportation in one. In this study, a new preservation strategy different from the cryopreservation or lyophilization was developed by reducing sEV particles aggregation.

Methods: The sEV were encapsulated in thermoresponsive gelatin methacryloyl (GelMA) hydrogels at $4^{\circ} \mathrm{C}$ to reduce particles aggregation during the reversible cross-linking process. The sEV movement was visualized in different mediums and particles' number, size, structure and protein of 28 days preserved sEV were compared to fresh sEV. Human umbilical vein endothelial cells (HUVEC) and rat adipose-derived stromal stem cells (rASC) were isolated and cultured with fresh and preserved sEV to test the cellular response. A mice subcutaneous model was adopted to detect controlled release and angiogenesis ability of preserved sEV.

Results: Through particles tracks visualization, GelMA hydrogels significantly decreased the sEV movement. After 28 days preservation in GelMA at $4^{\circ} \mathrm{C}$, the particles number, size, structure and protein of sEV were similar to fresh sEV. In vitro, preserved sEV had the same ability to promote cell proliferation, migration and angiogenesis as fresh sEV. In vivo, preserved sEV-GelMA could artificially regulate the absorptivity of GelMA hydrogels and controlled released $\mathrm{sEV}$ for therapeutic application, and preserved $\mathrm{sEV}$ encapsulated in GelMA significantly promoted angiogenesis in mice.

Conclusion: Our results demonstrated that SEV encapsulated in GelMA could be a novel strategy for long-term preservation of sEV for therapeutic application.

Keywords: small extracellular vesicles, particle track visualization, preservation, controlled release, aggregation, gelatin methacryloyl hydrogel

\section{Introduction}

Small extracellular vesicles (sEV) are small vesicles with a natural nanoscale phospholipid bilayer structure secreted by living cells. sEV are considered to be a natural carrier for the delivery of components such as proteins, lipids and nucleic acids from donor cells to recipient cells, thus enabling the cellular communication over different distances. ${ }^{1}$ sEV are widely used in tissue regeneration, ${ }^{2}$ targeted drug delivery, ${ }^{3}$ biological therapy and liquid biopsy, ${ }^{4,5}$ and have a very broad application prospect. 
For therapeutic applications, the effective preservation of isolated sEV has become an issue. SEV have been preserved by several major preservation methods including cryopreservation, lyophilization and spray drying. ${ }^{6-10}$ Not surprisingly, these methods, which are based on traditional cell cryopreservation or drug preservation, also have limitations, including dependence on low temperatures $\left(-20^{\circ} \mathrm{C},-80^{\circ} \mathrm{C}\right.$ or $\left.-196^{\circ} \mathrm{C}\right)$ and equipment, ${ }^{11}$ unfavorable transportation, ${ }^{12,13}$ complicated operation steps, ${ }^{12,13}$ unstable preservation effect, ${ }^{10}$ freeze-thawing circle damage $^{14}$ and difficult removal of cryoprotectants and lyoprotectants. ${ }^{7,15}$ Therefore, it is very important to find an efficient, convenient and low-cost method to preserve isolated sEV. sEV are membrane structured vesicles encapsulated by proteins, nucleic acids and lipids. ${ }^{16}$ At $4{ }^{\circ} \mathrm{C}$, the proteins, nucleic acids and lipids in the intact membrane structure are relatively stable. ${ }^{17,18}$ The preparation of sEV can be thought of as a colloid - a solution suspended with nanoscale dispersed particles, ${ }^{19} \mathrm{sEV}$ in solution do irregular Brownian transport and thus aggregate with each other, leading to the rupture of the membrane structure and the inactivation of its contents without the protection of the membrane structure. ${ }^{18,20-23}$ So the activity of sEV preserved in phosphate-buffered saline (PBS) decreases significantly after only a few days at $4^{\circ} \mathrm{C} \cdot{ }^{14,18,24}$ We hypothesized that if the aggregation of $\mathrm{SEV}$ could be reduced by limiting the movement of sEV, effective preservation of SEV could be achieved and the damage to sEV by the freeze-thaw circling process could be reduced as well.

Hydrogels are extremely hydrophilic gels with a threedimensional network structure. In recent years, many studies have found that loading hydrogels with SEV can be effective in sustaining the release of SEV in vivo. ${ }^{2,25-35}$ The sEV in the hydrogel is slowly released into the surrounding tissues over a long period of up to 1 month $^{33,34,36}$ and the sEV released from the hydrogels on different days still have the same structure. ${ }^{32}$ The pore size of many hydrogels after crosslinking is between $10-40 \mathrm{~nm}^{25,37}$ This can effectively limit the movement of sEV and meet our requirements for sEV preservation. Gelatin methacryloyl (GelMA) hydrogels are synthesized by grafting methacrylic anhydride (MA) onto gelatin molecular chains. ${ }^{25}$ It can undergo temperature-sensitive physical crosslinking (reversible) and ultraviolet (UV)-sensitive chemical crosslinking (irreversible) ${ }^{38}$ GelMA hydrogels are in a gel state at $4^{\circ} \mathrm{C}$, and gradually change to a liquid state at room temperature, Some of the basic properties of
GelMA hydrogels are very similar to natural extracellular matrix (ECM), making it biocompatible with sEV. GelMA hydrogels can also be microfabricated using different methodologies including micromolding, ${ }^{39,40}$ photomasking, ${ }^{41}$ bio-3Dprinting, ${ }^{42}$ selfassembly ${ }^{43}$ and microfluidic techniques ${ }^{44}$ to generate artificial designed constructs, which has a very broad application prospect. Recent studies have confirmed the efficacy of sEV loaded in GelMA hydrogels for the regeneration of osteochondral defects. ${ }^{36}$

As shown in Figure 1, this study encapsulated adipose tissue-derived sEV in GelMA hydrogels which limited the random movement of $\mathrm{sEV}$ and reduced the aggregation of $\mathrm{sEV}$, thus achieving long-term preservation of $\mathrm{sEV}$ at $4^{\circ} \mathrm{C}$, and experimentally confirmed that the preserved sEV by this method had similar therapeutic efficacy to fresh sEV. The preserved sEV-GelMA complex exhibited strong angiogenic ability and achieved controlled release of sEV in vivo by regulating UV-crosslinking time, showing great promise for therapeutic applications.

\section{Materials and Methods}

\section{Animals}

Experimental animals were purchased from Dashuo Experimental Animal Co. Ltd. (Chengdu, China). The care and use of the laboratory animals followed the guidelines of the Institutional Animal Care and Use Committee of West China School of Stomatology, Sichuan University. Animal experiments were reviewed and approved (WCHSIRB-D-2020-391) by the ethical review committee of the State Key Laboratory of Oral Diseases, West China School of Stomatology, Sichuan University. Experimental Animals were lived in a biology laboratory with suitable temperature (about $23 \pm 1{ }^{\circ} \mathrm{C}$ ), humidity (about $60-75 \%$ ) and a 12:12 light-dark cycle and plenty of fodder and clean water. All animal surgeries were performed under bioclean environment.

\section{Isolation of $s E V$}

Inguinal Fat pads from 4-week-old Sprague-Dawley rats were cut into $1 \mathrm{~mm}^{3}$ pieces and transferred to Celstir rotating bottle (Wheaton). After 2 days of cell culture, adipose tissue extracts (ATE) were prepared as described in our previous study. ${ }^{48}$ Next, ATE were filtered through $0.22 \mu \mathrm{m}$ filters (Millipore, USA), and then concentrated sequentially with Ultracel-3 membranes (Millipore, USA) and Ultracel-100 membrane (Millipore, USA) at $5000 \mathrm{~g}$, 


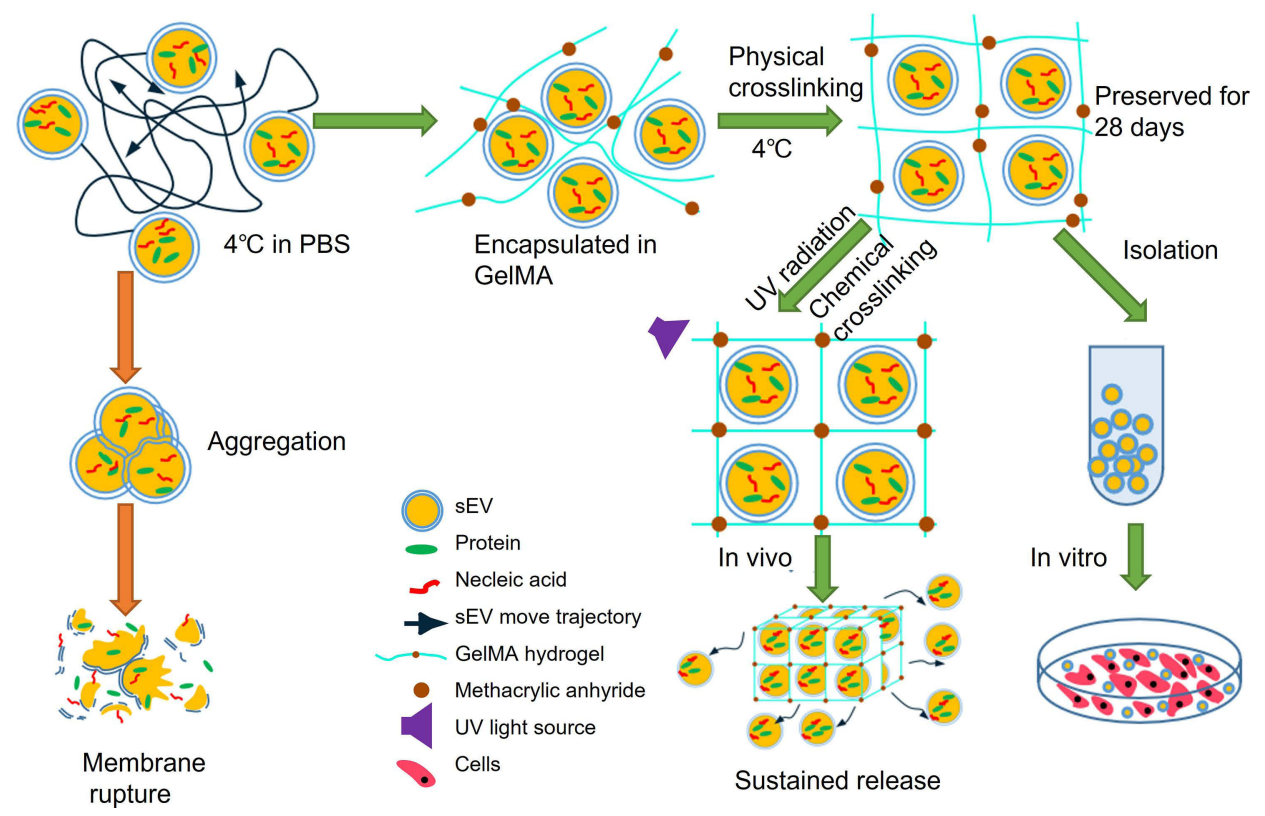

Figure I Schematic illustration of sEV preservation in GelMA hydrogels. sEV in PBS continuously move around in random patterns leads to particles aggregation, which resulted in membrane rupture (left). GelMA hydrogels could effectively limit the movement of $s E V$ and reduce the aggregation to effectively preserve $s E V$. The preserved $s E V$ were isolated for functional evaluation in vitro and UV-crosslinking sEV-GelMA complex system was adopted to compare the biological function of preserved sEV and fresh $s E V$ in vivo (right).

$4^{\circ} \mathrm{C}$ for $30 \mathrm{~min}$. Total Exosome Isolation ${ }^{\mathrm{TM}}$ reagent (Life Technologies) was added in the final solution according to the manufacturer's instructions to obtain fresh sEV.

\section{Differential Scanning Calorimetry}

Differential scanning calorimetry was performed to detect the pore size distribution of a hydrogel by performing calorimetry. Briefly, a 10mg hydrogel piece was placed in a sealable aluminium pan inside a differential scanning calorimeter (NETZSCH DSC 200F3). Sample temperature was adjusted to $-30^{\circ} \mathrm{C}$, held for $5 \mathrm{~min}$, then heated to $15^{\circ} \mathrm{C}$ held for $5 \mathrm{~min}$ and then cooled again to $-30^{\circ} \mathrm{C}$, with the temperature varying at a rate of $5^{\circ} \mathrm{C} \min ^{-1}$. The pore size distribution was calculated from $\Delta \mathrm{V} / \Delta \mathrm{Rp}$, where $\mathrm{Rp}$ is the pore radius. ${ }^{37,45}$

\section{Preservation of $s E V$}

In this study, several methods were selected to preserve sEV. After washing, (i) the sEV particles were carefully resuspended in $\mathrm{PBS}$, and stored at $4{ }^{\circ} \mathrm{C}$, (ii) the sEV particles was carefully resuspended in PBS containing $10 \%$ Dimethyl sulfoxide (DMSO), aliquoting $1 \mathrm{~mL}$ into each cryopreservation tube, wrapping tubes in heat isolated materials and storing in a $-80^{\circ} \mathrm{C}$ freezer overnight (about $16 \mathrm{~h}$ ), and then remove the heat isolated materials, (iii) the sEV particles was carefully resuspended in $5 \%, 10 \%$,
$15 \%, 20 \%(w / v)$ GelMA hydrogel (EFL) and $0.25 \%(w /$ v) lithium acylphosphinate photo-initiator (EFL) at 28$35^{\circ} \mathrm{C}$, keep in dark place and stored at $4^{\circ} \mathrm{C}$. The preservation concentration of sEV was $1 \times 10^{9}$ particles $\mu \mathrm{L}^{-1}$. When it was necessary to isolate the sEV again, the sEV preserved at $-80^{\circ} \mathrm{C}$ were thawed on ice for $30 \mathrm{~min}$ or $1-2 \mathrm{~min}$ at $37^{\circ} \mathrm{C}$ washed once in $20 \mathrm{~mL}$ PBS, and mixed with Total Exosome Isolation ${ }^{\mathrm{TM}}$ reagent (Life Technologies), the next isolation steps were performed as shown above. As for sEV encapsulated in GelMA hydrogel, the gel needs to be reheated to liquid state $\left(28-35^{\circ} \mathrm{C}\right)$, add 1 microliter of GelMA lysate (EFL) per milliliter and then add the Total Exosome Isolation ${ }^{\mathrm{TM}}$ Reagent. The following steps are described in the previous section.

\section{D sEV Particle Tracking}

Isolated sEV were labeled by carbocyanine dye $\mathrm{DiO}$ (life tech, USA) according to the instructions for in vivo visualization. DiO-labeled sEV were encapsulated in Gelma hydrogels, placed on dishes of coverslip thickness (MatTek), and imaged at $\times 60$ with immersion oil using a confocal microscopy (Olympus FV1000). Green fluorescence channel $1024 \times 1024 \times 256$ pixel images and videos were collected with $0.1 \mu \mathrm{m}$ spacing using the regular imaging mode. After $30 \mathrm{~min}, 30$ stacks were acquired. After acquisition, using the IMARIS X64 9.3.0 
(Bitplane) 'Spots' function to simulate particles tracks. The velocity of the particle is calculated by IMARIS software.

\section{Western Blot}

Proteins from 30ug $\left(1 \times 10^{10}\right.$ particles $)$ of $\mathrm{sEV}$ were detected with antibodies against CD9 (Zen Bioscience, 220642) and CD81 (ZenBioscience, 381296). Samples with the same initial sEV particle number were stored for 0,14 and 28 days using different methods.

\section{Nanoparticle Tracking Analysis (NTA)}

Fresh sEV were stored at $4^{\circ} \mathrm{C}$ in PBS, $4^{\circ} \mathrm{C}$ in $10 \%$ GelMA hydrogels, or $-80^{\circ} \mathrm{C}$ in $10 \% \mathrm{DMSO}$, respectively. Particles distribution and concentration of sEV were analyzed fresh or after storage (4, 7, 10, 14, 21 and 28 days) using NTA (Particle Metrix' ZetaView) and the corresponding software ZetaView 8.04.02. Prepare PBS, 10\%DMSO and 10\% GelMA hydrogels samples separately, store them for different days and the number of particles was calculated. Preserved sEV particles minus the stored media particles were the true particle numbers of sEV particles during preservation. NTA measurements were recorded and analyzed at 11 positions at $25^{\circ} \mathrm{C}$. The ZetaView machine was normalized using homogeneous $100 \mathrm{~nm}$ polystyrene particles.

\section{Transmission Electron Microscope (TEM)}

Fresh sEV and sEV that had been stored for 28 days at $4^{\circ} \mathrm{C}$ in PBS, $4^{\circ} \mathrm{C}$ in $10 \%$ GelMA hydrogel, or $-80^{\circ} \mathrm{C}$ in $10 \%$ DMSO for 28 days were dropped onto copper grids coated with film and stained with $2 \%$ phosphotungstic acid. Images were captured using an electron microscope (FEI).

\section{In vitro Fluorescent Imaging Assay}

sEV with the same initial particle number $\left(1 \times 10^{10}\right.$ particles) were stored for different days (0, 14, 28 days) under different storage conditions then labeled with $\mathrm{DiO}$ dye (Sigma Aldrich) according to the instruction. Fresh sEV with gradient particle numbers were labeled with $\mathrm{DiO}$ in the same way. The fluorescence value of DiO-labeled sEV were analyzed with a Meastro EX pro in vivo imaging system (PerkinElmer, USA) and linear correlation between the sEV particles and the DIO signals were examined.

\section{Cell Isolation and Culture}

The inguinal fat pads of SD rats were cut into $1 \mathrm{~mm}^{3}$ pieces, and digested with collagenase for $30 \mathrm{~min}$. The tissue was washed with PBS and centrifuged at
$1000 \mathrm{rpm}$ for $5 \mathrm{~min}$, and the remaining pellet was cultured in $\alpha$-modified Eagle's medium ( $\alpha$ - MEM; HyClone), $10 \%$ fetal bovine serum (FBS; Gibco) with penicillin streptomycin. rASCs were cultured at $37^{\circ} \mathrm{C}$ in $5 \% \mathrm{CO} 2$, and cell passaging was performed when monolayers of adherent cells reached $90 \%$ confluence. Human umbilical cords were obtained from the Department of Obstetrics of the West China Second University Hospital of Sichuan University. The approval number is WCHSIRB-D-2021015. We got the informed consent signed by the parents according to the Declaration of Helsinki. Collected umbilical cords were washed twice with phosphate buffered saline. In order to isolate HUVECs, the veins were filled with $0.2 \%$ collagenase and maintained for $30 \mathrm{~min}$ in a humidified atmosphere at $37^{\circ} \mathrm{C}$ and $5 \% \mathrm{CO} 2$ for 30 minutes. HUVECs were then obtained and cultured in ECM (ScienCell, USA).

\section{Cell Proliferation Assay}

HUVECs were seeded at a density of $8 \times 10^{2}$ cells per well and replaced after 1 day with ECM $(100 \mu \mathrm{L})$ containing 0 $\mathrm{sEV}, 4 \times 10^{10}$ fresh sEV particles, $4 \times 10^{10} \mathrm{sEV}$ particles preserved by GelMA hydrogel respectively. Cell number was assessed by the cell-counting kit-8 (CCK8, KeyGEN BioTECH, China). Proliferation curves were calculated from the absorbance values $(n=3)$. Then, samples were divided into 3 groups: (i) Blank: HUVECs cultured with ECM, (ii) Fresh sEV: HUVECs cultured with ECM and fresh sEV, (iii) GelMA sEV: HUVECs cultured with ECM and sEV store in GelMA hydrogel. The proliferation curve of rASCs was detected by the same method.

\section{Cell Migration Assay}

Cell migration assays were performed with $8.0 \mu \mathrm{m}$ Pore Polycarbonate Membrane Insert Transwell ${ }^{\circledR}$ (Corning). HUVECs were seeded with 20,000 cells per sample in the upper chamber and $600 \mu \mathrm{L}$ of medium divided into groups as described above was added to the lower chamber. After 18 hours, HUVECs were fixed with 4\% paraformaldehyde and stained with crystal violet staining solution. Migrated cells were counted in three randomly selected regions and repeated 3 times. The same method was used for the rASCs migration assay.

\section{Scratch Migration Assay}

HUVECs were exposed to starvation overnight and a uniform cell-free area zone was made with $200 \mu \mathrm{L}$ sterile plastic yellow tips. The movement and migration of cells 
to the cell-free zone were collected and monitored for 12 hours. The number of cells moving into the scratched area was counted using Image $J$ analysis software $(\mathrm{NIH}$, Bethesda, MD) for quantitative analysis. The same method was used in the rASCs scratch migration assay.

\section{Tube Formation Assay}

8000-cells-HUVECs in $50 \mu \mathrm{L}$ of ECM medium were added onto angiogenesis u-slide (Ibidi, Gräfelfing) covered with 10 ul Matrigel (Corning), and groups were divided as above, after $4 \mathrm{~h}$, images were taken with a microscope (Olympus, TH4-200). The total length of the tubes and nodes were calculated using Image $\mathrm{J}$ software.

\section{qRT-PCR}

HUVECs were cultured to $90 \%$ confluence in 6-well tissue plates, and then the cells culture media was culture medium was replaced and cells were collected from 4 days of culture. The PCR results were analyzed using the $2^{-\Delta \Delta C T}$ method and normalized to the GAPDH. Primers could be found in Supplementary Table S1.

\section{Evaluation of Rheological Properties}

GelMA hydrogels of $1 \mathrm{~mm}$ thickness with different degrees of crosslinking were prepared $0 \mathrm{~s}, 3 \mathrm{~s}$ and $5 \mathrm{~s}$ crosslinking on $\mathrm{C} 57 \mathrm{BL} / 6 \mathrm{~J}$ mouse skin with a visible light source of $405 \mathrm{~nm}$. The TA rheometer (TA-AR2000ex) was used to analyze the rheological properties of 3 groups of $10 \%$ GelMA hydrogel at $30^{\circ} \mathrm{C}$, the including the storage modulus $\left(\mathrm{G}^{\prime}\right)$, loss modulus $\left(\mathrm{G}^{\prime \prime}\right)$, and viscosity under different conditions (time, oscillation strain, and shear rate).

\section{In vivo Fluorescence Analysis}

The back of $\mathrm{C} 57 \mathrm{BL} / 6 \mathrm{~J}$ mice was unhaired, a subcutaneous incision was made in the center of the back, a $12 \times 2 \mathrm{~mm}$ silicone tube was implanted and the incision was sutured. Two sets of mixed materials, ie, Dio-labeled sEV encapsulated in non-fluorescent GelMA hydrogel and non-fluorescent sEV encapsulated in Red fluorescent-labeled GelMA(EFL), were injected into silicone tubes, and the GelMA hydrogels were $\mathrm{UV}$-crosslinked for $0 \mathrm{~s}, 3 \mathrm{~s}$ and $5 \mathrm{~s}$, respectively, on a layer of mice skin. Then the mice were imaged using the Meastro EX pro in vivo imaging system (PerkinElmer) to immediately image the mice. Bioluminescence imaging (BLI) signals from day 0 to day 6 were calculated by averaging the signals from ROIs.

\section{In vivo Angiogenesis Assay}

As mentioned in in vivo fluorescence analysis, $12 \times 2 \mathrm{~mm}$ silicone tubes were implanted subcutaneously in C57BL/ $6 \mathrm{~J}$ mice under anesthesia. The injections were divided into 4 groups, (i) $60 \mathrm{ul} 10 \%$ preserved GelMA hydrogels (ii) $8 \times 10^{10}$ fresh sEV capsulated in 60 ul $10 \%$ preserved GelMA hydrogels (iii) $8 \times 10^{10}$ initial particle number sEV capsulated in $60 \mu \mathrm{L} 10 \% 60 \mu \mathrm{L}$ GelMA hydrogels preserved at $4^{\circ} \mathrm{C}$ for 28 days (iv) $8 \times 10^{10}$ initial particle number sEV preserved in $30 \mu \mathrm{L}$ PBS at $4{ }^{\circ} \mathrm{C}$ for 28 day capsulated in 30ul 20\% preserved GelMA hydrogels. Six days after the injections, all mice were killed by Ethics Committee's approval. After skin removal, the digital photographs were taken with Image $J$ software to measure area of neovascularization. Next, Hematoxylin and Eosin (H\&E) staining and CD31 immunochemical staining were used to assess of angiogenesis.

\section{Statistical Analysis}

Results were expressed as mean \pm SD. Significant differences between two groups were calculated by one-way analysis of variance (ANOVA) test with Turkey posthoc test. Significant differences between repeated measurement data were calculated by repeated measurement data analysis of variance (MANOVA). $\mathrm{P}<0.05$ was considered statistically significant. Linear correlation analysis was used for correlation analysis. $\mathrm{R}>0.5$ was considered strong correlation. Statistical calculations were carried out using GraphPad Prism 6.0.

\section{Results}

\section{GelMA Hydrogels Reduced sEV}

\section{Movement}

The pore size of hydrogels with different concentrations of GelMA or sEV was measured by thermal method, and we found that the average pore size (Figure 2A) and pore size distribution (Supplementary Figure S1) of 5-20\% hydrogels did not change much. The pore size of the hydrogels increased with increasing sEV concentration, but remained less than $30 \mathrm{~nm}$. Next, we examined the variations of two types of crosslinking of hydrogels encapsulated with sEV $\left(1 \times 10^{9}\right.$ particles $\left.\mu \mathrm{L}^{-1}\right)$ with different hydrogel concentrations (Supplementary Figure 


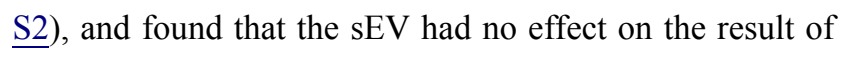
low-temperature physical cross-linking and UVcrosslinking.

By confocal microscopy observation and software analysis, we visualized the motion of sEV in different mediums. The sEV was found to undergo a fast irregular Brownian motion in PBS (Figure 2B; Supplementary Figure S3, Supplementary Video 1) while the sEV encapsulated in hydrogels underwent a significantly slower motion (Figure 2B, Supplementary Figure S3, Supplementary Video 2), with a significantly smaller extent and trajectory than the motion of the sEV in PBS at the same time (Supplementary Video 3, left particle: sEV motion in GelMA. right particle: sEV motion in PBS. Putting two particles in the same space). We analyzed the motion of single particles (Figure 2C) and obtained the same results. Quantitative analysis of specific particle velocities showed that sEVs in PBS moved more than 40 times faster than that in GelMA hydrogels (Figure 2D), suggesting that the $10 \%$ GelMA hydrogels effectively limited the irregular motion of sEV.

\section{sEV Encapsulated in GelMA Hydrogels}

Retained Their Particle Number, Size,

\section{Structure and Protein}

sEV were preserved for up to 4 weeks and $-80^{\circ} \mathrm{C}$ cryopreservation with $10 \%$ dimethyl sulfoxide (DMSO) was added as a positive control (PC). The results of nanoparticles tracking analysis (Figure $3 \mathrm{~A}$ ) showed that both $-80^{\circ} \mathrm{C}$ cryopreservation (PC) and $4^{\circ} \mathrm{C}$ GelMA hydrogel preservation $\left(4^{\circ} \mathrm{C}\right.$-GelMA) were effective in keeping the number of sEV particles from decreasing significantly. During a 4-week preservation period, the number of sEV particles decreased by approximately $20 \%$. In contrast, the number of sEV was significantly reduced under conventional $4^{\circ} \mathrm{C}$ preservation $\left(4^{\circ} \mathrm{C}-\mathrm{PBS}\right)$, dropping by half at around 10 days, which is consistent with some reported results. ${ }^{6,7} \mathrm{We}$ further performed a correlation between particle number and fluorescence values by $\mathrm{DiO}-$ labeled sEV (Supplementary Figure S4), and then quantified the fluorescence of preserved sEV (Figure $3 \mathrm{~B}$ and C), and confirmed similar conclusions. Thus, GelMA hydrogels could effectively maintain the particle number of sEV.

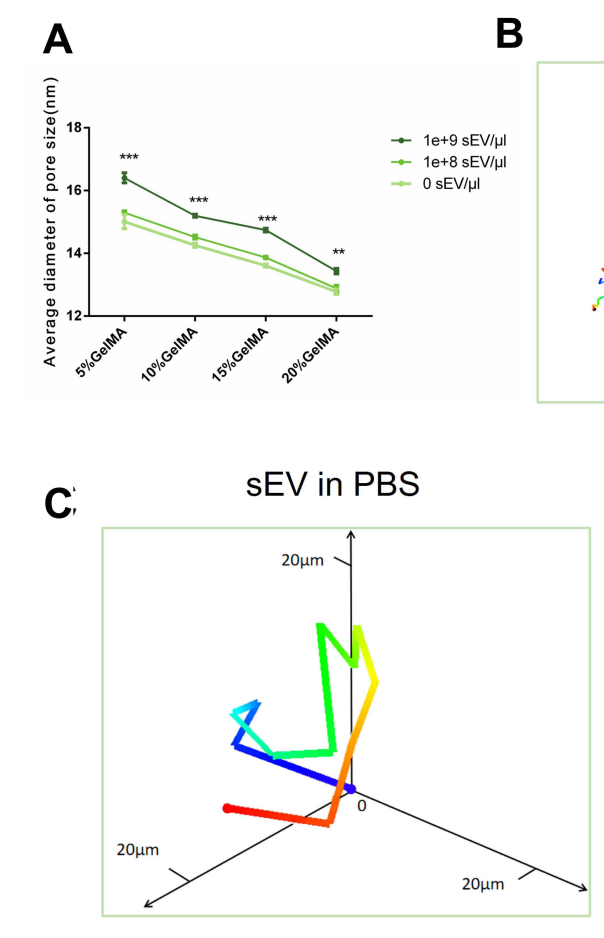

B sEV in PBS sEV in $10 \%$ GelMA
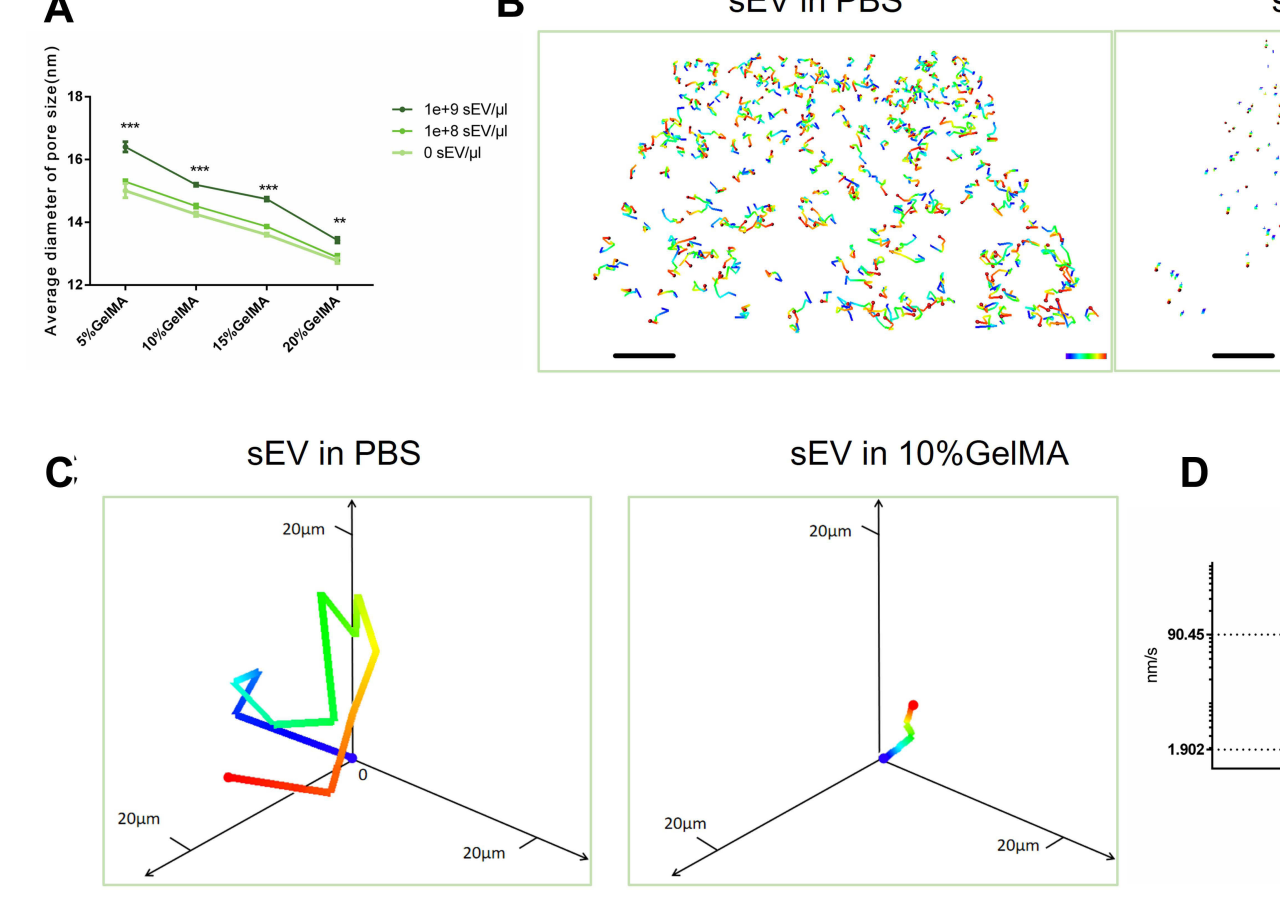

\section{SEV in $10 \%$ GeIMA}
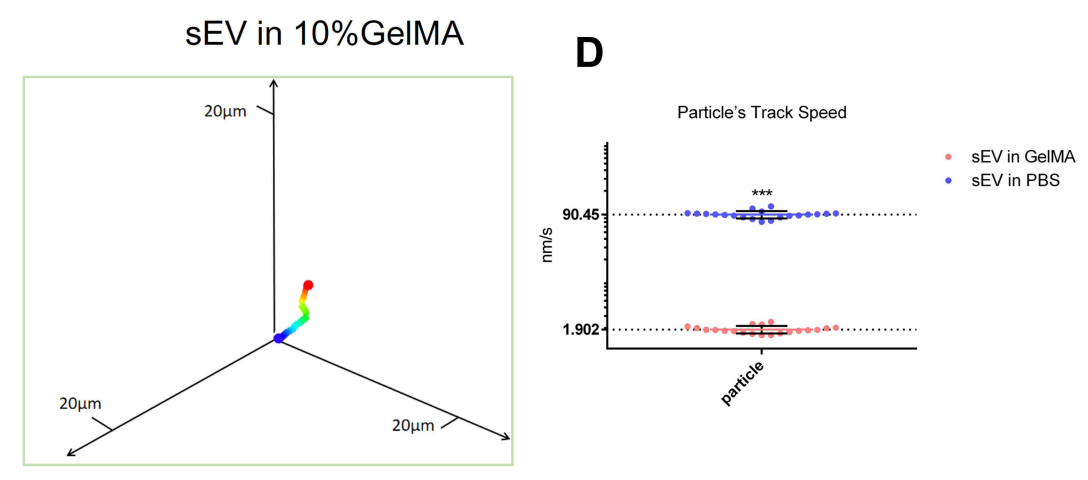

Time

Figure 2 Analysis of $s E V$ movement in GelMA hydrogels. (A) The pore size of GelMA hydrogels mixed with or without $s E V, n=3$ each group (B) The tracks of $s E V$ in PBS or $10 \% \mathrm{GelMA}$ hydrogels during same time period at $4^{\circ} \mathrm{C}$, Scale bar $=40 \mu \mathrm{m}$. (C) Single particle track analyzed in 3D coordinate system. (D) The particles tracks speed of sEV in PBS or 10\%GelMA hydrogels, $n=20$. The significance $(\mathbf{A}$ and $\mathbf{D})$ was tested with one-way ANOVA with Tukey posthoc test. $(* * p<0.01, * * * p<0.001)$. 
A

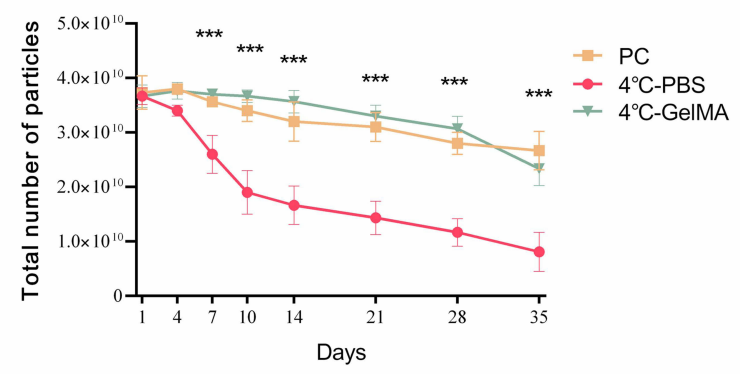

C

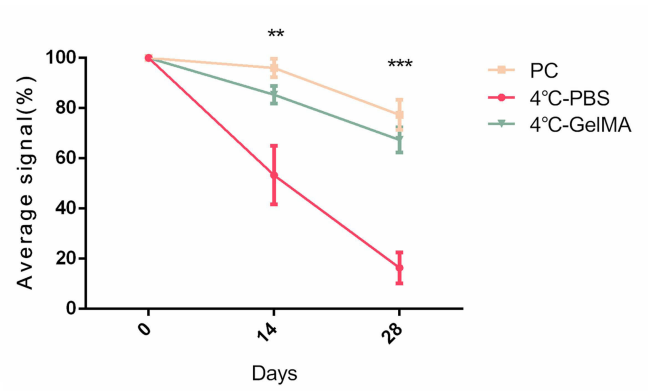

B

Od

14d

$28 d$

PC

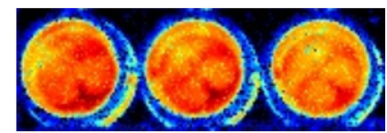

$4^{\circ} \mathrm{C}-$

PBS

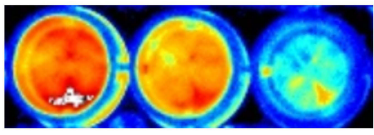

$4^{\circ} \mathrm{C}-$

Gelma

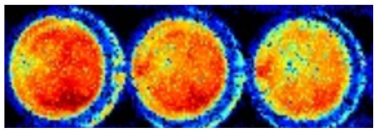

Free DiO

D

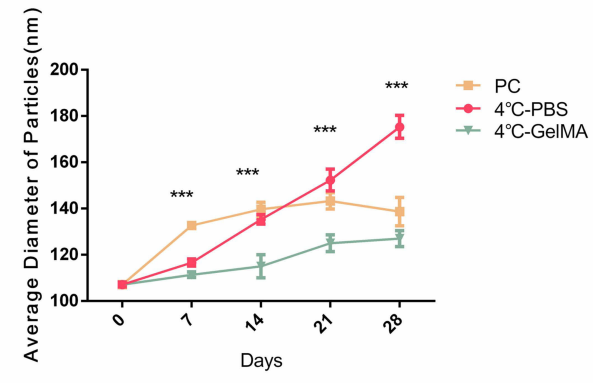

E

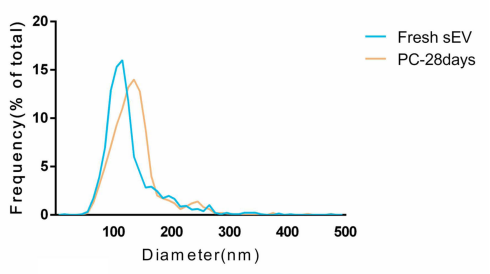

F

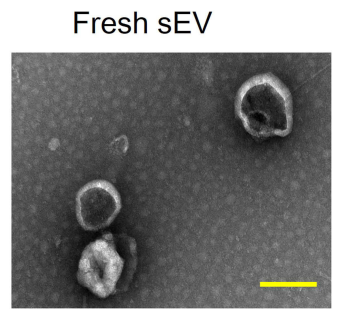

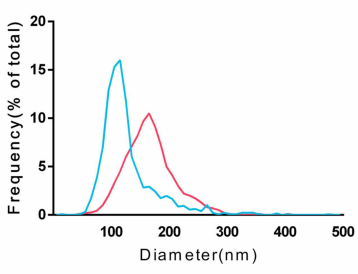

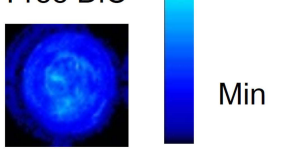

Min

Max

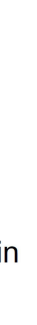

$$
\text { . }
$$


We also investigated the changes in SEV particle size after different preservation time. We found that the particle size increased significantly with time under $4^{\circ} \mathrm{C}$ PBS preservation (Figure 3D), which is also consistent with the previous findings. ${ }^{8,12,46}$ In terms of particle size distribution, the peak became larger and the proportion decreased, and the proportion of vesicles with large particle size (200-300 nm) increased, indicating that aggregation of sEV occurred. In contrast, the particle size of sEV stored in $-80^{\circ} \mathrm{C}$ freezing and hydrogels increased slightly, and the change in particle size distribution was not significant (Figure 3E).

As shown by the results of transmission electron microscopy (Figure 3F), fresh sEV had the standard sEV structure of round vesicles surrounded by a double layer of membrane. Conventionally preserved $\mathrm{sEV}$ at $4^{\circ} \mathrm{C}$ showed distinct sEV aggregation, with disrupted of vesicle structure and unclear boundaries between vesicles. In contrast, the vesicle structure of $\mathrm{sEV}$ preserved at $-80^{\circ} \mathrm{C}$ freezing and $4^{\circ} \mathrm{C}$ Gelma hydrogels was relatively normal and indistinguishable from that of fresh sEV. Combined with the results of transmission electron microscopy, the sEV preserved at $4^{\circ} \mathrm{C}-\mathrm{PBS}$ in our study showed significant aggregation, but the phenomenon was not observed in $4^{\circ} \mathrm{C}$-GelMA and PC.

In addition, we found that sEV stably expressed some marker proteins but with the increasing preservation days. As reported in some studies, SEV protein expression decreased significantly when stored at $4^{\circ} \mathrm{C}$ (Figure 3G), ${ }^{11}$ whereas sEV frozen at $-80^{\circ} \mathrm{C}(\mathrm{PC})$ and those stored in GelMA hydrogels at $4^{\circ} \mathrm{C}$ did not change significantly, so we have good reasons to believe that the SEV proteins stored in GelMA hydrogels were not changed.

\section{Preserved sEV Have Strong Ability to Promote Cells Proliferation, Migration}

\section{and Angiogenesis}

The sEV particle isolation rate under different mediums was investigated and the effective isolation rate (Figure 4A) of sEV in GelMA hydrogels was about $70 \%$, which was sufficient for conducting cell experiments. In the cell proliferation assay, cell migration assay and scratch assay, the promotion effect of GelMA hydrogelspreserved sEV for 28days (GelMA-sEV-28Days) compared with fresh sEV (Fresh sEV) was basically the same for human Umbilical Vein endothelial cells (HUVEC) (Figure 4B-D) and rat adipose stem cells
(rASCs) (Supplementary Figure S5A-C). GelMA hydrogel-preserved sEVs also exhibited a pro-angiogenic capacity relatively consistent with that of fresh SEVs in tube formation assays (Figure 4E) and angiogenesis-related gene PCR assays (Figure 4F). These results were highly satisfactory and fully demonstrated the efficiency and utility of our preservation method.

\section{Preserved sEV-GelMA Complex Achieved sEV Controlled Release in vivo}

Here, we treated 28 days of preserved $1 \times 10^{9}$ particles $\mu \mathrm{L}^{-1}$ sEV in $10 \%$ Gelma hydrogels as a complex and investigated the sustained and controlled release effect of this complex. The results showed different UV-crosslinking duration of GelMA Hydrogels could alter the properties of the GelMA-sEV complex properties. A general view showed that GelMA-sEV without UV-crosslinking complex were viscous liquid with some surface tension When the crosslinking time reached 3 seconds, the complex was partially solidified and poorly supported. When the crosslinking time reached 5 seconds, the complex was completely solidified and could remain in the shape of the circular mould (Figure 5A). Rheological test showed that the viscosities, storage modulus and loss modulus of GelMA-sEV complex changed obviously with different crosslinking time (Figure 5B and C). We then performed in vivo experiments in which silicone tubes with 28days preserved GelMA-sEV complexes were implanted into in the subcutaneous layer of the dorsum of C57BL/6J mice back (Figure 5D), and found that the fluorescence values of the red fluorescently labeled hydrogel alone (Figure 5E) changed very similarly to those of the DiO-labeled sEV wrapped in GelMA hydrogel alone (Figure 5F) during 0-6 days. The difference in the change in gel absorbance rate and $\mathrm{sEV}$ release rate from $0 \mathrm{~s}$ to $5 \mathrm{~s}$ of $\mathrm{UV}$ crosslinking was more than 5-fold (Figure 5G). We found the absorption rate of hydrogel is highly correlated with SEV release rate in vivo. Therefore, the absorption rate of the hydrogels in vivo could be controlled by controlling the degree of UV-crosslinking, which indirectly controls the release of $\mathrm{sEV}$, resulting in an artificially controlled sEV-GelMA release system.

\section{Preserved sEV Promoted Angiogenesis in vivo}

The pro-angiogenic effect of sEV preserved in GelMA hydrogels could be clearly observed after 6 days of 
A

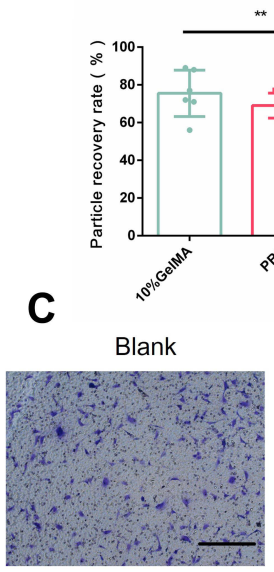

D
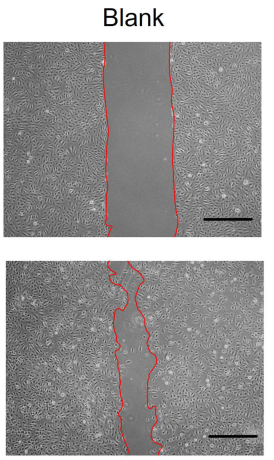

E

Blank

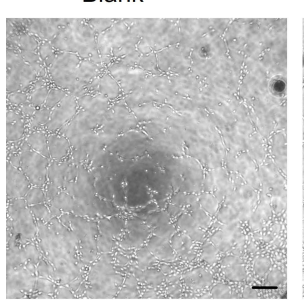

F
B

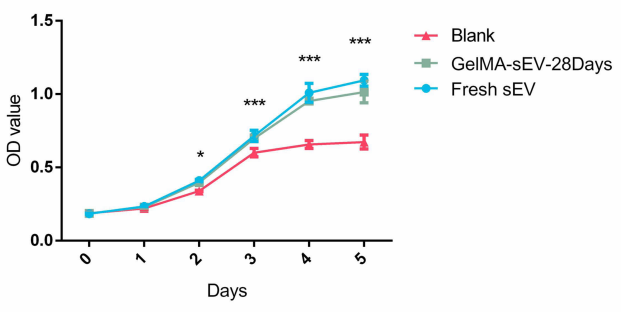

Gelma-sEV-28days

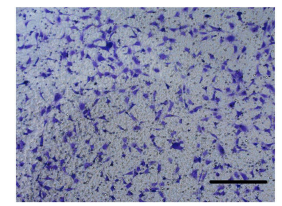

Gelma-sEV-28days
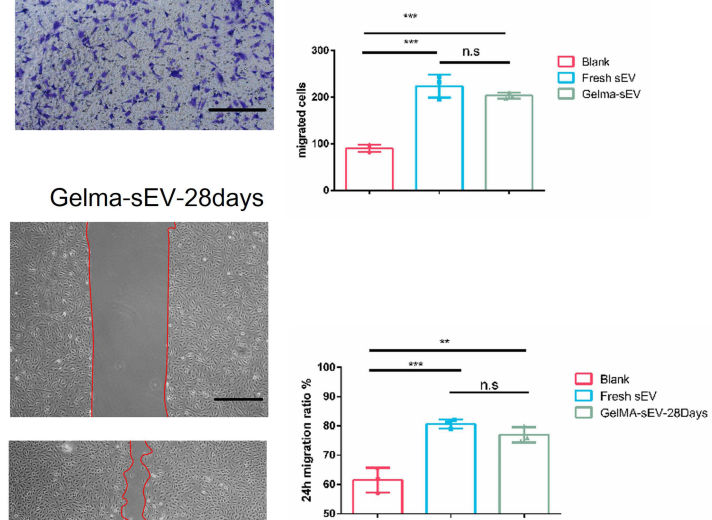

Fresh sEV
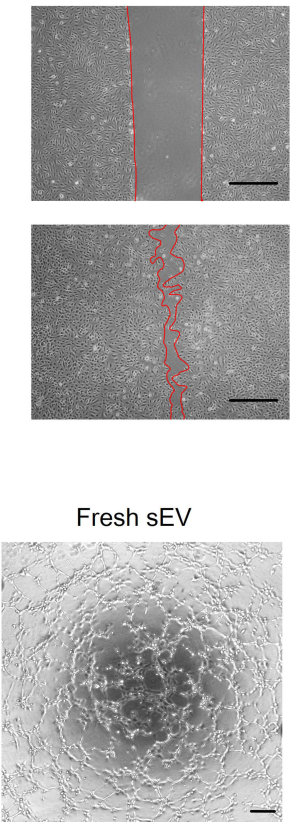

Gelma-sEV-28days
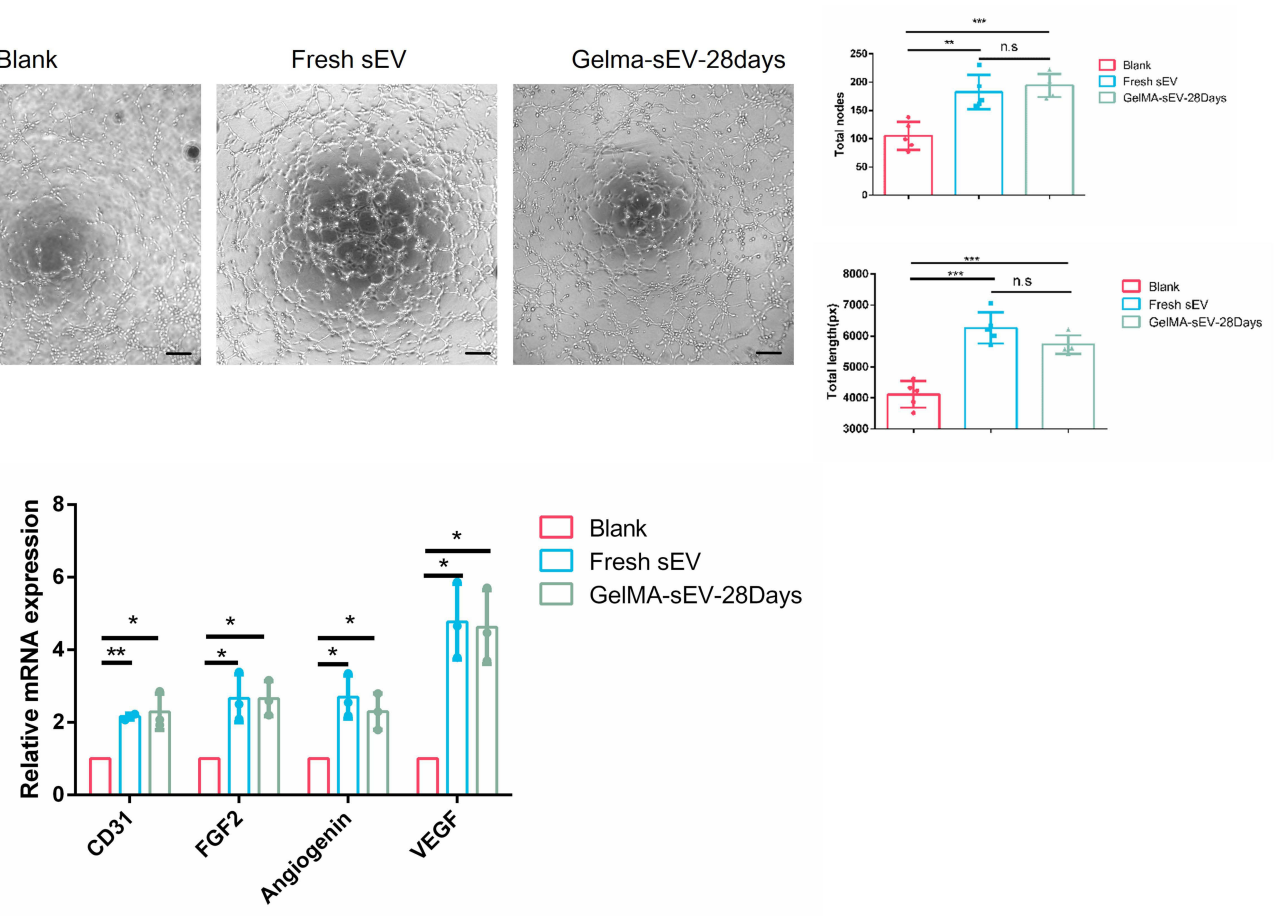

Figure 4 The effect of preserved $s E V$ on HUVEC proliferation, migration, tube formation and angiogenesis differentiation. (A) Recovery rate of sEV from different preservation method by TEl isolation. (B) Proliferation of HUVEC co-cultured with fresh sEV and sEV preserved in GelMA hydrogels for 28 days. $n=3$ for each group. (C) Images of migrated HUVECs in different group. Scale bar $=200 \mu \mathrm{m} . \mathrm{n}=3$ for each group. (D) Images of scratch assay in different group. Scale bar $=100 \mu \mathrm{m}$. Time transition of the percentage of cell-free zone against initial scratch area after 12 hour, $n=3$ for each group. (E) Tube-like structures of HUVECs in different group. Scale bar $=200 \mu \mathrm{m}$. $n=$ 3 for each group. (F) The expression of angiogenesis markers (VEGF, FGF2, CD31, Angiogenin) was detected by qRT-PCR at 4 days post sEV treatment, $n=3$ for each group. The significance $(\mathbf{A}-\mathbf{E})$ was tested with one-way ANOVA with Tukey posthoc test. $\left({ }^{*} \mathrm{p}<0.05,{ }^{* *} \mathrm{p}<0.01,{ }^{*} *{ }^{*} \mathrm{p}<0.00 \mathrm{I}\right)$. 
A

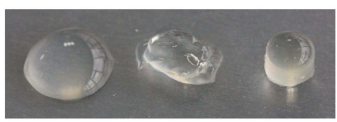

UV-crosslinking time $\quad 0 S \quad 3 S \quad 5 S$

C

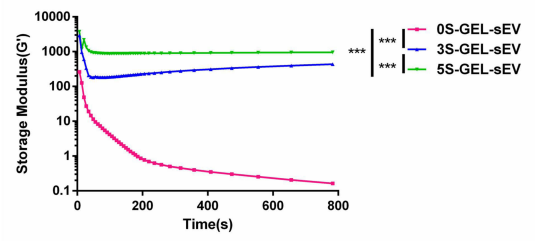

B
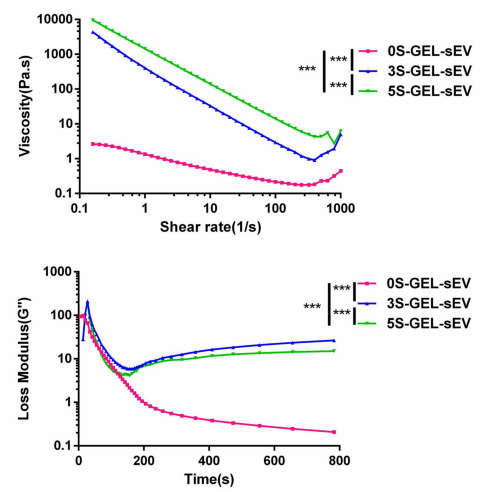

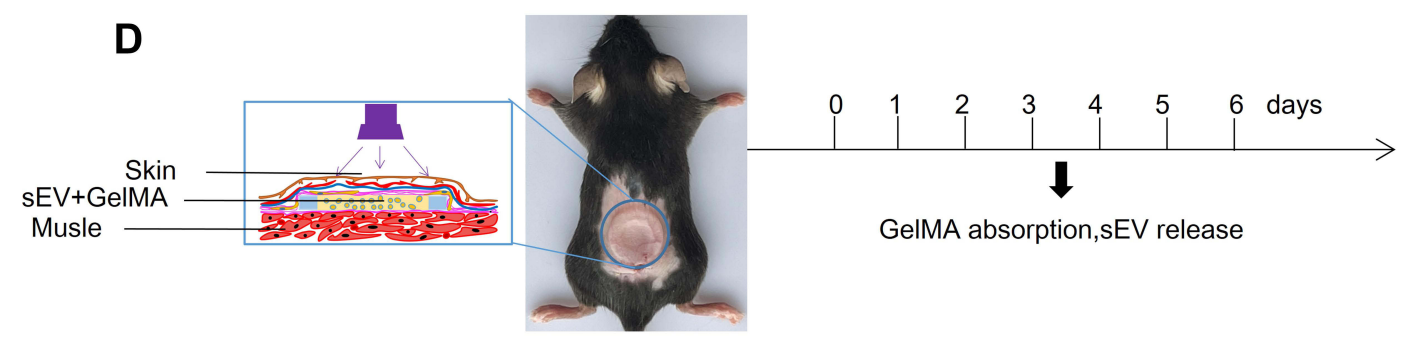

$\mathbf{E}$

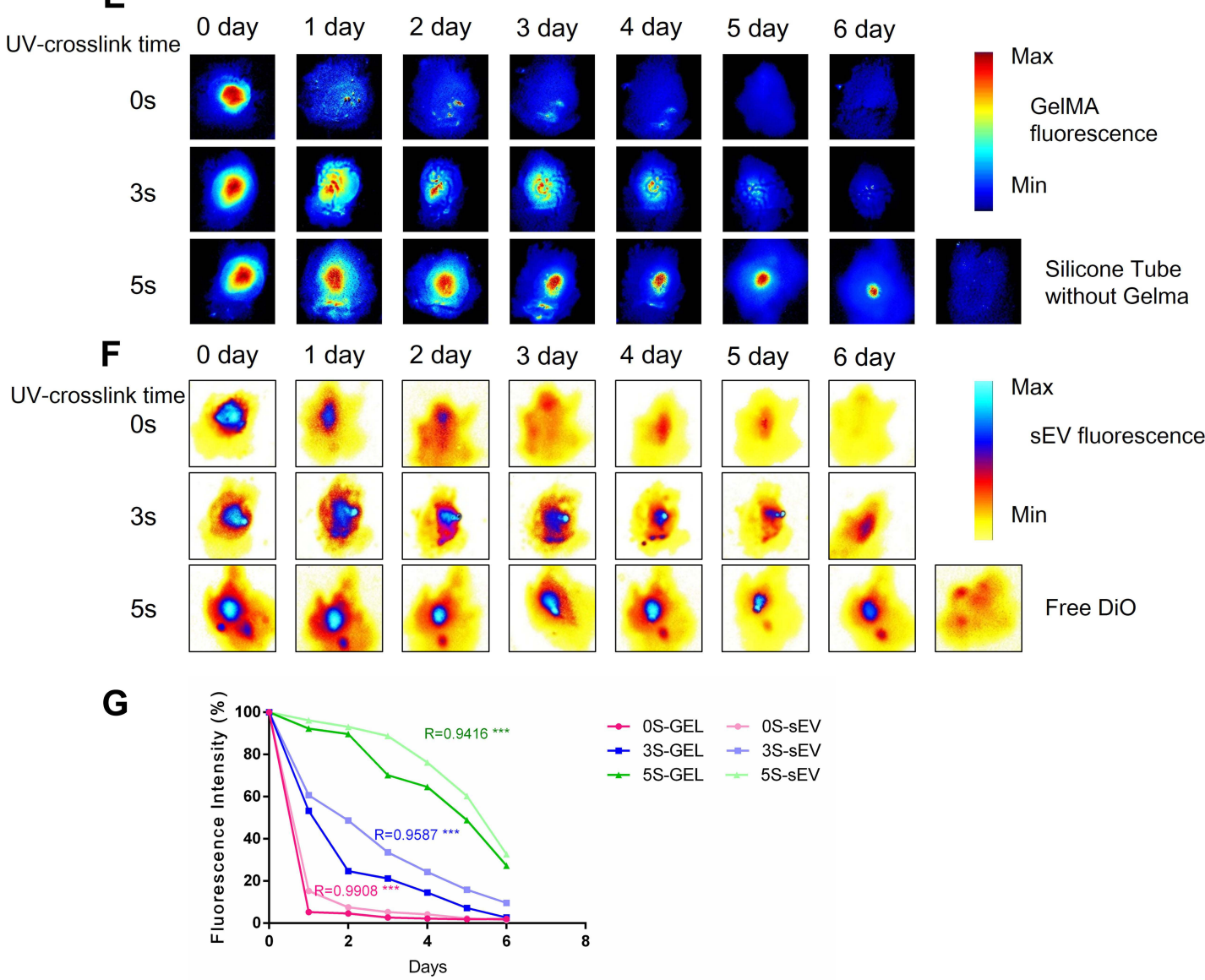

Figure $5 \mathrm{sEV}$ could be controlled released from sEV-GelMA complex after preservation in vivo. (A) General view of different UV-crosslinking time for preserved GelMA-sEV complex (B) Viscosity of different UV-crosslinking time GelMA-sEV complex dressing with the shear rate from 0.1 to I 0 I/s. (C) Rheological behavior of different UV-crosslinking time GelMA-sEV complex was evaluated via rheometer. The significance was tested with repeated measurement data analysis of variance (MANOVA). (D) General view and schematic view of the experimental operation in vivo. (E) Ex vivo visualization of red fluorescent labeled GelMA hydrogels UV-crosslinked by different time period for 6 days. (F) Ex vivo visualization of DiO labeled sEV encapsulated in GelMA hydrogels UV-crosslinked by different time period for 6 days. (G) Quantification analysis of the fluorescence intensity for red fluorescent labeled GelMA hydrogels and DiO labeled sEV with different crosslinking time. The correlation coefficient $\mathrm{R}$ and significance of Figure $5 \mathrm{G}$ was calculated by linear correlation analysis. $(* * * p<0.001)$ 
subcutaneous implantation in silicone tubes by injection of sEV-hydrogels systems with different preservation methods and days. The pro-angiogenic effect of sEV preserved in hydrogels (GelMA-sEV-28days) was consistent with that of fresh sEV (Fresh sEV), with uniform distribution and well-defined neovascular borders, whereas the proangiogenic effect of sEV preserved in PBS for 28 days (PBS-sEV-28days) effect was slightly worse, but also clearly distinguished from the negative control group (NC) (Figure 6A and B). In HE-stained sections, neovascularization of the fascial layer, filled was evident in the Fresh sEV and GelMA-sEV-28days groups, filled with a large number of erythrocytes, whereas in the PBS-Sev -28days group had fewer vessels, their diameters were thinner (Figure 6C). In the immunohistochemically stained sections, it was observed that there were more vascular endothelial cells expressing CD31 positivity in the Fresh sEV and GelMA-sEV-28days groups, whereas there were fewer vascular endothelial cells expressing CD31 positivity in the PBS-sEV-28 days group and almost no vascular endothelial cells expressing CD31 positivity were seen in the NC group (Figure 6D). Thus, we concluded that the GelMA hydrogels preserved SEV have the ability to promote angiogenesis in vivo consistent with fresh sEV.

\section{Discussions}

In recent years, $\mathrm{sEV}$ have been widely used in medicine, but the preservation of sEV still face with many challenges. At present, there is no preservation method which combines high recovery rate, low cost, convenience, and easy-transportation in one. In order to solve the problem of $\mathrm{sEV}$ aggregates in $4^{\circ} \mathrm{C}$, leading to membrane rupture and reduced activity, we designed a new sEV preservation strategy by encapsulating sEV in GelMA hydrogel to reduce aggregation, thus achieving the goal of preserving $\mathrm{sEV}$.

In order to select a suitable GelMA hydrogel concentration, we first examined the pore size of hydrogels with different GelMA or sEV concentrations. Scanning differential thermal method is a classic way to measure the pore size of hydrogels. ${ }^{37,45}$ The pore size of GelMA hydrogels is reported to be inversely proportional to the concentration, our results showed same trend. ${ }^{38}$ The pore size of GelMA hydrogels was significantly smaller than the pore size of $\mathrm{sEV}$, thus limiting the movement of sEV.

As described in many studies, loading sEV in hydrogels had little effects on the basic properties of the hydrogels. $^{25,33,47}$ Considering that the preserved GelMA and sEV might be used directly in vivo, we chose a concentration of $1 \times 10^{9}$ particles $\mu \mathrm{L}^{-1} \mathrm{sEV}$ and $10 \%$ GelMA hydrogels, which is a common concentration for tissue regeneration. ${ }^{36,40,48,49}$ Recent studies considered that adipose tissue could produce and secreted a wide range of mediators regulating adipose tissue and important distant targets as an endocrine organ. ${ }^{50}$ Adipose tissuederived sEV are easily accessible and have been shown to promote lipogenesis, angiogenesis differentiation and immunomodulation. ${ }^{4,51-54}$ There are many isolation methods for sEV, and polyethylene glycol (PEG) precipitation is a rapid, convenient and high yield isolation method that widely used for the isolation of large amounts of $\mathrm{sEV}{ }^{55} \mathrm{~A}$ recent study showed that the sEV obtained by PEG precipitation had the same high purity as that isolated by ultracentrifugation. ${ }^{56}$ Therefore PEG precipitation was chosen to isolate sEV in our study. It is worth mentioning that $\mathrm{sEV}$ isolated by collagenase is ${ }^{57}$ not suitable for preservation by this method because the residual collagenase will enzymatically dissolve the GelMA hydrogels.

According to previous studies, hydrogels loaded with $\mathrm{sEV}$ can have a sustained release effect in vivo for more than one month, and the released sEV remains physiologically functional. ${ }^{33,34,36}$ Some studies have found that the released $\mathrm{sEV}$ at any time still have the same structure as fresh $\mathrm{sEV}^{32}$ However, as mentioned above, sEV will be deactivated in a few days in an environment of $4^{\circ} \mathrm{C}$, and the body temperature is much higher than $4^{\circ} \mathrm{C}$, which seems to indicate that hydrogels have a certain protective effect on $\mathrm{sEV}$, but the exact mechanism is unknown. Based on our experimental results, it was confirmed that the movement speed of sEV encapsulated in GelMA hydrogels was significantly slowed down, mainly due to the limitations of GelMA hydrogels, which was consistent with our initial speculation.

Currently, the most common preservation methods of sEV are cryopreservation and lyophilization. Cryopreservation is a preservation strategy that reduces the temperature below the temperature that is required for biological reactions to maintain the functional stability and is usually applied at $\mathrm{t}-20^{\circ} \mathrm{C},-80^{\circ} \mathrm{C}$ and $-196^{\circ} \mathrm{C}$. Studies have shown that the rate of protein degradation for $-20^{\circ} \mathrm{C}$ preservation is greater than that for $-80^{\circ} \mathrm{C}$ preservation. ${ }^{15}$ The degradation rate of sEV proteins such as ALIX, HSP70 and TSG101 decreased and the degradation rate at $-80^{\circ} \mathrm{C}$ was less than that at $-20^{\circ} \mathrm{C} .{ }^{14}$ However, there was no significant difference between -196 and $-80^{\circ} \mathrm{C}$ cryopreservation. ${ }^{23}$ The cryopreservation method 
A
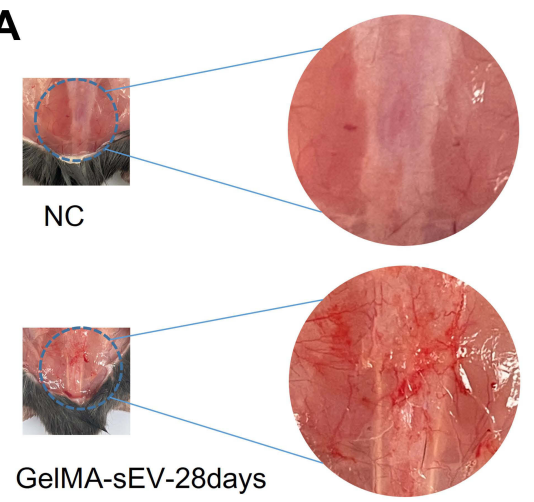
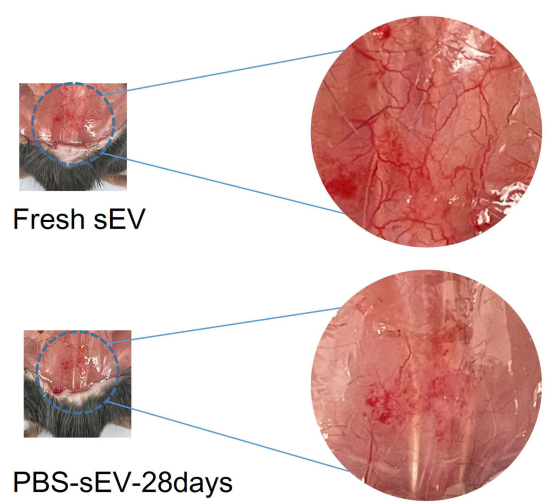

B
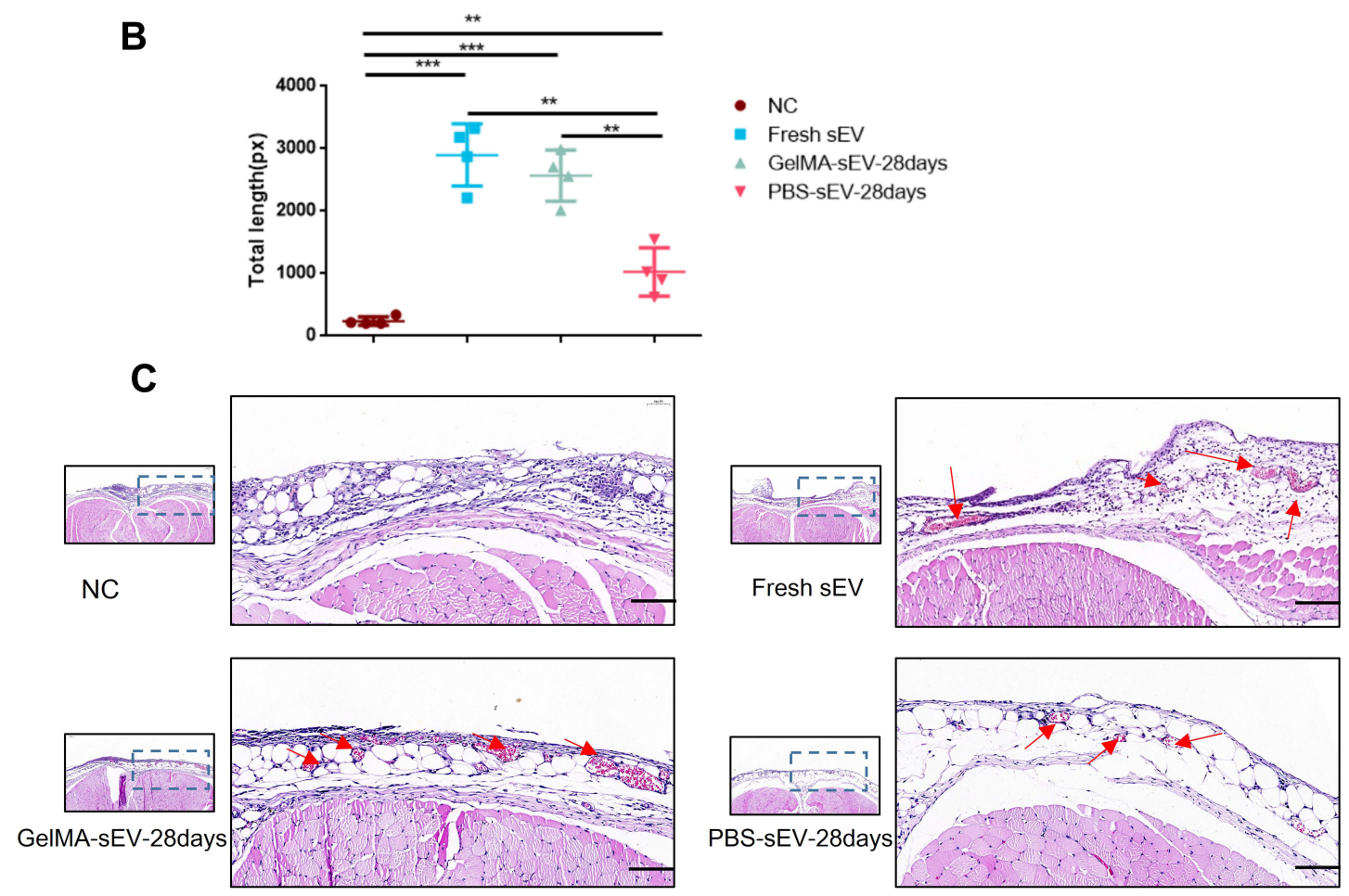

D

NC

Fresh sEV

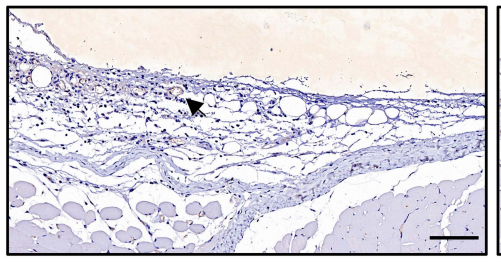

GelMA-sEV-28days
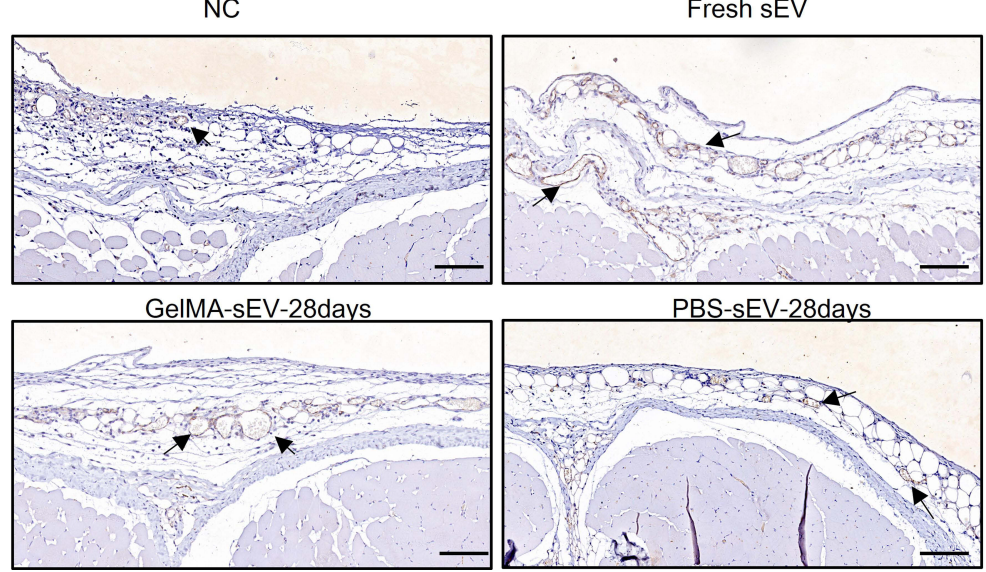

PBS-sEV-28days

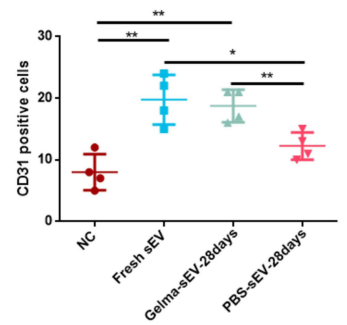

Figure 6 Preserved GelMA-sEV complex promoted angiogenesis in the subcutaneous fascial layer of C57BL/6) mice. (A) General view of angiogenesis in the subcutaneous fascial layer in different groups on 6th day after treatment.NC, negative control. (B) Total length of new vessels in the fascial layer from each groups, $n=4$. (C) Representative HE-stained images of angiogenesis in the subcutaneous fascial layer (red arrows: new vessels with erythrocytes) Scale bar $=50 \mu \mathrm{m}$. (D) $I \mathrm{mmunohistochemical}$ staining of CD3I-positive endothelial cells in each groups was used to visualize vessels. CD3I-positive endothelial cells were quantified from 5 independent visions per sample (black arrows: $C D 3$ I positive endothelial cells) Scale bar $=50 \mu \mathrm{m}, \mathrm{n}=4$. The significance $(\mathbf{A}$ and $\mathbf{D})$ was tested with one-way ANOVA with Tukey posthoc test. ( ${ }^{\mathrm{p}}<$ $0.05, * * \mathrm{p}<0.01, * * * \mathrm{p}<0.001)$ 
is likely to "frostbite". The "frostbite" described here is mostly related to the imbalance of osmosis pressure during the freezing process and the formation of ice crystals within the biological particles. ${ }^{7}$ To avoid the phenomenon of frostbite, some appropriate concentrations cryoprotectants are often used to increase the preservation time. Permeability and non-permeability cryoprotectant are used to protect the membrane structure. Some research suggested that dimethyl sulfoxide (DMSO) could be used as a permeability cryoprotectant to help maintain the morphology of these vesicles for long-term storage although DMSO was unable to preserve the morphology of all vesicles in the sample. ${ }^{12}$ Despite this cryopreservation had some negative reports, ${ }^{23,24}$ it was still widely used. Lyophilization also need lyoprotectants such as trehalose and polyvinylpyrrolidone 40 to stabilize the membrane structure. ${ }^{58}$ Considering the convenience of operation, we chose DMSO cryopreservation as a positive control (PC), strictly followed standard cryopreservation and thawing procedures. $^{12}$

The change of the particle size of $\mathrm{sEV}$ is of great significance for preservation. Generally, there are two reasons for the increase of the particle size of $\mathrm{sEV}$. One is aggregation, which has been shown that aggregation will increase in a few days at $4^{\circ} \mathrm{C} .^{6}$ The size distribution of multiple overlapping Gaussian size distributions led to the appearance of long-tailed mixtures, suggesting that freezing produced a population of larger nanovesicle aggregates up to $400 \mathrm{~nm}$ in diameter, ${ }^{18}$ In addition, after the surface treatment of sEV, the vesicles have difficulty contacting each other and causing aggregation. It was observed that the particle size could remain constant for about 1 month. ${ }^{22}$ Another scenario is the size expansion of individual vesicle, which is mainly caused by the hypotonic environment. ${ }^{7}$ In addition, smaller vesicle sizes have also been reported, which are mainly fragments resulting from vesicle rupture. ${ }^{12,46}$ Our results showed that the particles' number, size, structure and protein of 28-day-preserved $\mathrm{sEV}$ were similar to those of fresh $\mathrm{sEV}$, this preservation method avoids the cryogenic process, greatly preserves the structure and number of sEV, and has the advantage of being low-cost, convenient, and transportable.

Protective agents should be added during cryopreservation, lyophilization as mentioned above. In previous sEV preservation study, the preserved SEV were never reisolated for functional validation, and residual protectants (DMSO, trehalose, albumin or polyvinylpyrrolidone 40) were not suitable for cellular experiments. ${ }^{59}$ The relatively low efficiency of re-isolation of SEV in DMSO is relatively low through PEG precipitation, which may be an important reason limiting the widespread use of this preservation method. Even though the function of sEV preserved in DMSO at $-80^{\circ} \mathrm{C}$, can be consistent with fresh $\mathrm{sEV}$ of the same particle number, the difference in the reisolation efficiency of sEVs from different preservations $(>20 \%)$ made it unnecessary to test the function of sEV preserved in DMSO at $-80^{\circ} \mathrm{C}$. A high isolation rate ensures the efficiency of application, and our preservation method had a relatively high isolation rate of $75 \%$.

In studies related to SEV preservation, physical crosslinking of GelMA hydrogels occurs at low temperatures, and the gels is reversible and can be converted to a liquid state at room temperature for sEV re-isolation and subsequent use. The chemical UV-crosslinking of GelMA Hydrogels could be used to form irreversible gels for in vivo application. The treatment and tissue regeneration of many diseases require long-term drug administration such as wound healing, ${ }^{60}$ cardiac repair, ${ }^{61}$ spinal cord injury, ${ }^{32}$ osteochondral defect ${ }^{25}$ and kidney repair. ${ }^{62}$ The half-life of sEV in vivo is very short, and they are metabolized by the liver within a few hours. ${ }^{63}$ Sustained release of $\mathrm{sEV}$ is required in many cases in order to reduce the trauma and inconvenience associated with repeated dosing. We found that the 28 days preserved sEV-GelMA complex system could achieve the sustained release as well as controlled release of sEV in vivo. The crosslinking degree of the hydrogels is controlled by the crosslinking time. We observed a change in the rheological properties of the hydrogels, and a change in the in vivo absorption, which is consistent with previous reports. ${ }^{64}$ When the GelMA hydrogels were in liquid state, sEV could move freely in the hydrogels and extracellular matrix and will be quickly absorbed by the surrounding tissues. Since absorption rate of the gel is also very fast at same time, the absorption rate of the gel is close to the release of sEV In our study, after 5 seconds of UV-crosslinking we found that the release rate of sEV remained essentially the same as the absorption rate of hydrogels, which is inconsistent with the results of many studies. ${ }^{25,36}$ In some studies the release rate of $\mathrm{SEV}$ was usually tested changes in the number of by SEV particles or protein changes in the PBS under in vitro conditions. ${ }^{2,33,65}$ In reality, in vivo hydrogels do not swell and the pore size does not change much, and it is difficult for sEV to be released into the surrounding tissues by their own movement. The main factor determining the release of sEV comes from the absorption of the hydrogels itself. It is 
worth mentioning that some specially designed hydrogels could control the crosslinking degree of the gel in vitro or in vivo, allowing it to be cleaved after cross-linking, such as light-triggerable hydrogels, ${ }^{47}$ matrix metalloproteinase-2 sensitive self-assembling peptide hydrogels, ${ }^{66} \mathrm{PH}$ sensitiveself-assembling peptidehydrogels, ${ }^{34}$ which could also achieve a relatively precise controlled release effect in vitro. Our experiments fully confirmed the relationship between gel absorption and sEV release and laying the groundwork for the design of future studies on the sustained and controlled release of $\mathrm{sEV}$ through the hydrogels-sEV complex system.

After determining the relationship between sEV release and GelMA hydrogel absorption, we designed an in vivo experiment with a relatively short duration and significant effects. First, silicone tubes were placed in the subcutaneous fascia layer of C57BL/6J mice, and then the samples including sEV-GelMA complex were injected into the silicone tube. The sEV-GelMA complex were UV-crosslinked for 3 seconds, and the samples were collected and observed 6 days later. The area of the dorsal subcutaneous fascial layer was chosen because there are no superficial natural blood vessels here, which is very convenient for comparative study. According to previous studies, sEV from adipose tissue was highly effective in promoting angiogenesis. ${ }^{48,54}$ Therefore, the ability to promote angiogenesis suitable for testing the function of preserved sEV. Our results demonstrated that GelMA had no effect on angiogenesis, whereas the ability of preserved sEV to promote angiogenesis was still evident.

GelMA hydrogels have been widely used in various medical applications due to its suitable biological properties and tunable physical characteristics. ${ }^{38}$ It has good biocompatibility as it has similar properties are similar to native extracellular matrix (ECM). We found the following advantages of GelMA hydrogels for preservation, (i): the presence of temperature-sensitive reversible physical cross-linking facilitates sEV loading and isolation. (ii): the possibility of irreversible UV-crosslinking action in vivo. (iii): good biocompatibility, administered together without affecting sEV activity. (iv): mature preparation technology, which allows synthesis or purchase of manufactured products. Due to the good compatibility between sEV and GelMA hydrogels, this therapeutic combination can be widely used for the treatment and tissue regeneration by the methods described above. In addition to the effective preservation of $\mathrm{SEV}$ and controlled release of $\mathrm{sEV}$, the preserved sEV-GelMA complex constructed by us also have very promising applications in vivo.

\section{Conclusion}

Based on the therapeutic potential of sEV in the context of future clinical application, we developed an efficient, low-cost, convenient, high isolation rate and transportable strategy of $\mathrm{sEV}$ preservation at $4^{\circ} \mathrm{C}$ by encapsulating sEV in GelMA hydrogels to limit the movement of sEV, and reduce sEV aggregation. The results indicated that sEV preserved in GelMA hydrogels for 28 days in $4^{\circ} \mathrm{C}$ had similar particle number, size and characteristics as fresh sEV. The preserved sEV could be isolated subsequently and promoted cell proliferation, migration and differentiation in the same degree as fresh sEV. In vivo we observed the 28 days-preserved sEV still has a strong ability to promote angiogenesis. In addition, we investigated and constructed a preserved sEV-GelMA complex controlled release system, which is a trinity system of preservation-isolation- application.

\section{Acknowledgments}

This work was supported by National Key R\&D Program of China (2017YFA0104800) and Key Technology R\&D Program of Sichuan Province (2019YFS0312).

\section{Disclosure}

All authors reported no conflicts of interest in this work.

\section{References}

1. Tkach M, Thery C. Communication by extracellular vesicles: where we are and where we need to go. Cell. 2016;164(6):1226-1232. doi:10.1016/j.cell.2016.01.043

2. Zhang S, Yang Y, Jia S, et al. Exosome-like vesicles derived from Hertwig's epithelial root sheath cells promote the regeneration of dentin-pulp tissue. Theranostics. 2020;10(13):5914-5931. doi:10.7150/thno.43156

3. Wang J, Li G, Tu C, et al. High-throughput single-cell analysis of exosome mediated dual drugs delivery, in vivo fate and synergistic tumor therapy. Nanoscale. 2020;12(25):13742-13756. doi:10.1039/D0NR02344B

4. Yang XX, Sun C, Wang L, Guo XL. New insight into isolation, identification techniques and medical applications of exosomes. J Control Release. 2019;308:119-129. doi:10.1016/j.jconrel.2019.07.021

5. Kohaar I, Chen Y, Banerjee S, et al. A urine exosome gene expression panel distinguishes between indolent and aggressive prostate cancers at biopsy. J Urol. 2021;205(2):420-425. doi:10.1097/JU.0000000000001374

6. Zhang Y, Bi J, Huang J, Tang Y, Du S, Li P. Exosome: a review of its classification, isolation techniques, storage, diagnostic and targeted therapy applications. Int J Nanomedicine. 2020;15:6917-6934. doi:10.2147/IJN. S264498

7. Kusuma GD, Barabadi M, Tan JL, Morton DAV, Frith JE, Lim R. To protect and to preserve: novel preservation strategies for extracellular vesicles. Front Pharmacol. 2018;9:1199. doi:10.3389/fphar.2018.01199

8. Bosch S, de Beaurepaire L, Allard M, et al. Trehalose prevents aggregation of exosomes and cryodamage. Sci Rep. 2016;6:36162. doi: $10.1038 /$ srep36162

9. Munagala R, Aqil F, Jeyabalan J, Gupta RC. Bovine milk-derived exosomes for drug delivery. Cancer Lett. 2016;371(1):48-61. doi:10.1016/j.canlet.2015.10.020 
10. Wu JY, Li YJ, Hu XB, Huang S, Xiang DX. Preservation of small extracellular vesicles for functional analysis and therapeutic applications: a comparative evaluation of storage conditions. Drug Deliv. 2021;28(1):162-170. doi:10.1080/10717544.2020.1869866

11. Lee M, Ban -J-J, Im W, Kim M. Influence of storage condition on exosome recovery. Biotechnol Bioprocess Eng. 2016;21(2):299-304. doi:10.1007/s12257-015-0781-x

12. Wu Y, Deng W, Klinke DJ. Exosomes: improved methods to characterize their morphology, RNA content, and surface protein biomarkers. Analyst. 2015;140(19):6631-6642. doi:10.1039/C5A N00688K

13. Frank J, Richter M, de Rossi C, Lehr CM, Fuhrmann K, Fuhrmann G. Extracellular vesicles protect glucuronidase model enzymes during freeze-drying. Sci Rep. 2018;8(1):12377. doi:10.1038/s41598-01830786-y

14. Cheng YZQ, Han Q. Effect of $\mathrm{pH}$, temperature and freezing-thawing. pdf. Protein Cell. 2018;10(4):298-299.

15. Lorincz AM, Timar CI, Marosvari KA, et al. Effect of storage on physical and functional properties of extracellular vesicles derived from neutrophilic granulocytes. J Extracell Vesicles. 2014;3:25465. doi:10.3402/jev.v3.25465

16. Kalluri R, LeBleu VS. The biology, function, and biomedical applications of exosomes. Science. 2020;367:6478. doi:10.1126/science. aau6977

17. Jin Y, Chen K, Wang Z, et al. DNA in serum extracellular vesicles is stable under different storage conditions. BMC Cancer. 2016;16 (1):753. doi:10.1186/s12885-016-2783-2

18. Maroto R, Zhao Y, Jamaluddin M, et al. Effects of storage temperature on airway exosome integrity for diagnostic and functional analyses. J Extracell Vesicles. 2017;6(1):1359478. doi:10.1080/ 20013078.2017.1359478

19. Hood J, Scott M, Wickline SJ. Maximizing exosome colloidal stability following electroporation. Anal Bionanl Chem. 2014;448:41-49.

20. Evtushenko EG, Bagrov DV, Lazarev VN, Livshits MA, Khomyakova E. Adsorption of extracellular vesicles onto the tube walls during storage in solution. PLoS One. 2020;15(12):e0243738. doi:10.1371/journal.pone.0243738

21. Herrmann IK, Wood MJA, Fuhrmann G. Extracellular vesicles as a next-generation drug delivery platform. Nat Nanotechnol. 2021;16 (7):748-759. doi:10.1038/s41565-021-00931-2

22. Lathwal S, Yerneni SS, Boye S, et al. Engineering exosome polymer hybrids by atom transfer radical polymerization. Proc Natl Acad Sci $U S$ A. 2021;118(2):e2020241118. doi:10.1073/pnas.2020241118

23. Dong X, Li M, Li Q, et al. Effects of cryopreservation on microparticles concentration, procoagulant function, size distribution, and morphology. Med Sci Monit. 2019;25:6675-6690. doi:10.12659/ MSM.917962

24. Jeyaram A, Jay SJ. Preservation and storage stability of extracellular vesicles for therapeutic applications. AAPS J. 2017;20(1):1. doi:10.1208/s12248-017-0160-y

25. Chen P, Zheng L, Wang Y, et al. Desktop-stereolithography 3D printing of a radially oriented extracellular matrix/mesenchymal stem cell exosome bioink for osteochondral defect regeneration. Theranostics. 2019;9(9):2439-2459. doi:10.7150/thno.31017

26. Zhang K, Zhao X, Chen X, et al. Enhanced therapeutic effects of mesenchymal stem cell-derived exosomes with an injectable hydrogel for hindlimb ischemia treatment. ACS Appl Mater Interfaces. 2018;10(36):30081-30091. doi:10.1021/acsami.8b08449

27. Silva AKA, Perretta S, Perrod G, et al. Thermoresponsive gel embedded with adipose stem-cell-derived extracellular vesicles promotes esophageal fistula healing in a thermo-actuated delivery strategy. ACS Nano. 2018;12 (10):9800-9814. doi:10.1021/acsnano.8b00117

28. Hernandez MJ, Gaetani R, Pieters VM, et al. Decellularized extracellular matrix hydrogels as a delivery platform for microRNA and extracellular vesicle therapeutics. Adv Ther (Weinh). 2018;1(3). doi:10.1002/adtp.201800032
29. Shafei S, Khanmohammadi M, Heidari R, et al. Exosome loaded alginate hydrogel promotes tissue regeneration in full-thickness skin wounds: an in vivo study. J Biomed Mater Res A. 2020;108 (3):545-556. doi:10.1002/jbm.a.36835

30. $\mathrm{Xu} \mathrm{N}$, Wang L, Guan J, et al. Wound healing effects of a Curcuma zedoaria polysaccharide with platelet-rich plasma exosomes assembled on chitosan/silk hydrogel sponge in a diabetic rat model. Int J Biol Macromol. 2018;117:102-107. doi:10.1016/j.ijbiomac. 2018.05.066

31. Shi Q, Qian Z, Liu D, et al. GMSC-derived exosomes combined with a chitosan/silk hydrogel sponge accelerates wound healing in a diabetic rat skin defect model. Front Physiol. 2017;8:904. doi:10.3389/fphys.2017.00904

32. Li L, Zhang Y, Mu J, et al. Transplantation of human mesenchymal stem-cell-derived exosomes immobilized in an adhesive hydrogel for effective treatment of spinal cord injury. Nano Lett. 2020;20 (6):4298-4305. doi:10.1021/acs.nanolett.0c00929

33. Mardpour S, Ghanian MH, Sadeghi-abandansari H, et al. Hydrogelmediated sustained systemic delivery of mesenchymal stem cell-derived extracellular vesicles improves hepatic regeneration in chronic liver failure. ACS Appl Mater Interfaces. 2019;11 (41):37421-37433. doi:10.1021/acsami.9b10126

34. Mol EA, Lei Z, Roefs MT, et al. Injectable supramolecular ureidopyrimidinone hydrogels provide sustained release of extracellular vesicle therapeutics. Adv Healthc Mater. 2019;8(20):e1900847. doi:10.1002/adhm.201900847

35. Lin J, Wang Z, Huang J, et al. Microenvironment-protected exosome-hydrogel for facilitating endometrial regeneration, fertility restoration, and live birth of offspring. Small. 2021;17(11):e2007235. doi:10.1002/smll.202007235

36. Hu H, Dong L, Bu Z, et al. miR-23a-3p-abundant small extracellular vesicles released from Gelma/nanoclay hydrogel for cartilage regeneration. J Extracell Vesicles. 2020;9(1):1778883. doi:10.1080/ 20013078.2020.1778883

37. Lenzini S, Bargi R, Chung G, Shin JW. Matrix mechanics and water permeation regulate extracellular vesicle transport. Nat Nanotechnol. 2020;15(3):217-223. doi:10.1038/s41565-020-0636-2

38. Yue K, Trujillo-de Santiago G, Alvarez MM, Tamayol A, Annabi N, Khademhosseini A. Synthesis, properties, and biomedical applications of gelatin methacryloyl (GelMA) hydrogels. Biomaterials. 2015;73:254-271. doi:10.1016/j.biomaterials.2015. 08.045

39. Liu T, Weng W, Zhang Y, Sun X, Yang HJM. Applications of gelatin methacryloyl (GelMA) hydrogels in microfluidic technique-assisted tissue engineering. Molesules. 2020;25(22):5305.

40. Yuan Z, Yuan X, Zhao Y, et al. Injectable GelMA cryogel microspheres for modularized cell delivery and potential vascularized bone regeneration. Small. 2021;17(11):e2006596. doi:10.1002/smll.2020 06596

41. Nichol J, Koshy S, Bae H, Hwang C, Yamanlar S, Khademhosseini AJB. Cell-laden microengineered gelatin methacrylate hydrogels. Biomaterials. 2010;31(21):5536-5544. doi:10.1016/j. biomaterials.2010.03.064

42. Buyuksungur S, Hasirci V, Hasirci NJ. 3D printed hybrid bone constructs of PCL and dental pulp stem cells loaded GelMA. J Biomed Mater Res A. 2021;109:2425-2437. doi:10.1002/jbm.a.37235

43. Qi H, Ghodousi M, Du Y, et al. DNA-directed self-assembly of shape-controlled hydrogels. Nat Commun. 2013;4:2275. doi:10. 1038/ncomms3275

44. Ebrahimi M, Ostrovidov S, Salehi S, et al. Enhanced skeletal muscle formation on microfluidic spun gelatin methacryloyl (GelMA) fibres using surface patterning and agrin treatment. J Tissue Eng Regen Med. 2018;12(11):2151-2163. doi:10.1002/term.2738

45. Bousmina MI. Determination of pore size distribution for mesoporous materials and polymeric gels by means of DSC measurements: thermoporometry. Polymer. 2000;129:607-615. 
46. Sokolova V, Ludwig AK, Hornung S, et al. Characterisation of exosomes derived from human cells by nanoparticle tracking analysis and scanning electron microscopy. Colloids Surf B Biointerfaces. 2011;87(1):146-150. doi:10.1016/j.colsurfb.2011.05.013

47. Henriques-Antunes H, Cardoso RMS, Zonari A, et al. The kinetics of small extracellular vesicle delivery impacts skin tissue regeneration. ACS Nano. 2019;13(8):8694-8707. doi:10.1021/acsnano.9b00376

48. Dong J, Wu Y, Zhang Y, Yu M, Tian W. Comparison of the therapeutic effect of allogeneic and xenogeneic small extracellular vesicles in soft tissue repair. Int J Nanomed. 2020;15:6975-6991. doi:10.2147/IJN.S269069

49. Cuvellier M, Ezan F, Oliveira H, et al. 3D culture of HepaRG cells in GelMa and its application to bioprinting of a multicellular hepatic model. Biomaterials. 2021;269:120611. doi:10.1016/j.biomaterials.2020.120611

50. Ailhaud GJ. Adipose tissue as a secretory organ: from adipogenesis to the metabolic syndrome. $C R$ Biol. 2006;329(8):570-577;discussion 653-575. doi:10.1016/j.crvi.2005.12.012

51. Flaherty S, Grijalva A, Xu X, Ables E, Nomani A, Ferrante AJS. A lipase-independent pathway of lipid release and immune modulation by adipocytes. Science. 2019;363(6430):989-993. doi:10.1126/science. aaw2586

52. Dai M, Yu M, Zhang Y, Tian W. Exosome-like vesicles derived from adipose tissue provide biochemical cues for adipose tissue regeneration. Tissue Eng Part A. 2017;23(21-22):1221-1230. doi:10. 1089/ten.tea.2017.0045

53. Zhang Y, Yu M, Dai M, et al. miR-450a-5p within rat adipose tissue exosome-like vesicles promotes adipogenic differentiation by targeting WISP2. J Cell Sci. 2017;130(6):1158-1168. doi:10.1242/jcs.197764

54. Huang J, Wang L, Tian W. Small extracellular vesicles derived from adipose tissue prevent bisphosphonate-related osteonecrosis of the jaw by promoting angiogenesis. Int J Nanomed. 2021;16:3161-3172. doi:10.2147/IJN.S305361

55. Li M, Lou D, Chen J, et al. Deep dive on the proteome of salivary extracellular vesicles: comparison between ultracentrifugation and polymer-based precipitation isolation. Anal Bionanl Chem. 2021;413(2):365-375. doi:10.1007/s00216-020-03004-w

56. Kuang Y, Zheng X, Zhang L, et al. Adipose-derived mesenchymal stem cells reduce autophagy in stroke mice by extracellular vesicle transfer of miR-25. J Extracell Vesicles. 2020;10(1):e12024. doi:10.1002/jev2.12024
57. Crescitelli R, Lässer C, Lötvall JJ. Isolation and characterization of extracellular vesicle subpopulations from tissues. Nat Protoc. 2021;16(3):1548-1580. doi:10.1038/s41596-020-00466-1

58. El Baradie KBY, Nouh M, O'Brien Iii F, et al. Freeze-dried extracellular vesicles from adipose-derived stem cells prevent hypoxia-induced muscle cell injury. Front Cell Dev Biol. 2020;8: 181. doi:10.3389/fcell.2020.00181

59. Hebling J, Bianchi L, Basso F, et al. Cytotoxicity of dimethyl sulfoxide (DMSO) in direct contact with odontoblast-like cells. Dent Mater. 2015;31(4):399-405. doi:10.1016/j.dental.2015.01.007

60. Wang M, Wang C, Chen M, et al. Efficient angiogenesis-based diabetic wound healing/skin reconstruction through bioactive antibacterial adhesive ultraviolet shielding nanodressing with exosome release. ACS Nano. 2019;13(9):10279-10293. doi:10.1021/acsnano. $9 \mathrm{~b} 03656$

61. Han C, Zhou J, Liang C, et al. Human umbilical cord mesenchymal stem cell derived exosomes encapsulated in functional peptide hydrogels promote cardiac repair. Biomater Sci. 2019;7(7):2920-2933. doi:10.1039/C9BM00101H

62. Zhang C, Shang Y, Chen X, et al. Supramolecular nanofibers containing arginine-glycine-aspartate (RGD) peptides boost therapeutic efficacy of extracellular vesicles in kidney repair. ACS Nano. 2020;14 (9):12133-12147. doi:10.1021/acsnano.0c05681

63. Wang J, Chen D, Ho EA. Challenges in the development and establishment of exosome-based drug delivery systems. J Control Release. 2020;329:894-906.

64. Zheng K, Du D. Recent advances of hydrogel-based biomaterials for intervertebral disc tissue treatment: a literature review. J Tissue Eng Regen Med. 2021;15(4):299-321. doi:10.1002/term.3172

65. Liu X, Yang Y, Li Y, et al. Integration of stem cell-derived exosomes with in situ hydrogel glue as a promising tissue patch for articular cartilage regeneration. Nanoscale. 2017;9(13):4430-4438. doi:10.10 39/C7NR00352H

66. Zhou Y, Liu S, Zhao M, et al. Injectable extracellular vesicle-released self-assembling peptide nanofiber hydrogel as an enhanced cell-free therapy for tissue regeneration. J Control Release. 2019;316:93-104. doi:10.1016/j.jconrel.2019.11.003
International Journal of Nanomedicine

\section{Publish your work in this journal}

The International Journal of Nanomedicine is an international, peerreviewed journal focusing on the application of nanotechnology in diagnostics, therapeutics, and drug delivery systems throughout the biomedical field. This journal is indexed on PubMed Central, MedLine, CAS, SciSearch ${ }^{\circledR}$, Current Contents ${ }^{\mathbb{R}} /$ Clinical Medicine, $^{-}$

\section{Dovepress}

Journal Citation Reports/Science Edition, EMBase, Scopus and the Elsevier Bibliographic databases. The manuscript management system is completely online and includes a very quick and fair peer-review system, which is all easy to use. Visit http://www.dovepress.com/ testimonials.php to read real quotes from published authors. 